\title{
Propriedades Psicométricas da Escala Multidimensional de Suporte Social Percebido
}

\author{
Larissa Maria David Gabardo-Martins ${ }^{1}$ \\ Maria Cristina Ferreira \\ Felipe Valentini \\ Universidade Salgado de Oliveira, Niterói, RJ, Brasil
}

\begin{abstract}
Resumo
O suporte social pode ser definido como a percepção de que existem pessoas que proporcionam ao indivíduo recursos afetivos ou, até mesmo, financeiros. O suporte social percebido relaciona-se à percepção de que o suporte encontra-se disponível, caso o indivíduo dele necessite. O objetivo desta pesquisa é buscar evidências iniciais de validade dos escores da Escala Multidimensional de Suporte Social Percebido, no contexto brasileiro. Participaram do estudo 831 trabalhadores, de ambos os sexos, que responderam à versão inicial da escala, contendo 12 itens. As análises fatoriais evidenciaram que a versão brasileira manteve os itens e os fatores do instrumento original (família, amigos e outros significantes). As análises multigrupos atestaram a invariância configural, métrica e escalar entre os grupos divididos quanto ao gênero e à categoria ocupacional (profissionais das áreas exatas e humanas). Os dados obtidos na presente pesquisa permitiram a conclusão de que a escala é um instrumento com evidências de validade de estrutura, o que possibilita seu uso em situações futuras de pesquisa.
\end{abstract}

Palavras-chave: Redes sociais, escalas, validade estatística.

\section{Psychometric Properties of the Multidimensional Scale of Perceived Social Support}

\begin{abstract}
Social support can be defined as the perception that there are people who provide individuals with emotional or even financial resources. Perceived social support relates to the perception that support is available if the individual needs it. The present study's aim was to discover, within the Brazilian context, preliminary validity evidence as to the scores of the Multidimensional Scale of Perceived Social Support. The survey involved 831 workers of both sexes, who answered the preliminary version of the scale's questionnaire, containing 12 items. Factor analysis demonstrated that the Brazilian version preserved the original instrument's items and factors (family, friends and significant others). Multigroup analysis demonstrated configural, metric and scalar invariance between groups classified by sex and occupational category (professionals in the areas of exact sciences and humanities). The data we obtained in the present study led us to the conclusion that the scale is an instrument with evidence of internal structure validity, thus permitting its use in future research.
\end{abstract}

Keywords: Social networks, scaling, statistical validity.

Endereço para correspondência: Rua Sérvulo Mello, 56, Caju, Silva Jardim, Rio de Janeiro, RJ, Brasil 28820000. Fone: 22 99857-8802. E-mail: laragabardo@yahoo.com.br 


\section{Propiedades Psicométricas de la Escala Multidimensional de Apoyo Social Percibido}

\section{Resumen}

Apoyo social puede ser definido como la percepción de que hay personas que prestan los recursos emocionales, individuales, o incluso financieros. El apoyo social percibido se refiere a la percepción de que el apoyo está disponible, si el individuo necesita. El objetivo de esta investigación es buscar la evidencia inicial de validez de la Escala Multidimensional de Apoyo Social Percibido, en el contexto brasileño. El estudio incluyó a 831 trabajadores, de ambos sexos, que respondieron a la versión inicial de la escala, que contiene 12 ítems. Las análisis factoriales mostraron que la versión brasileña conservó los ítems y los factores del instrumento original (la familia, los amigos y otros significativos). Multigrupos análisis han atestiguado la invarianza configural, métrica y escalar entre los grupos divididos por género y categoría ocupacional (exactos y humanos profesionales). Los datos obtenidos en esta investigación llevaron a la conclusión de que la escala es un instrumento con evidencia de validez, lo que permite su uso en situaciones futuras.

Palabras clave: Redes sociales, escalas, validación estadística.

O suporte social diz respeito à percepção de que existem pessoas que proporcionam ao indivíduo recursos afetivos ou, até mesmo, financeiros, bem como à sensação de pertencimento e de acolhimento por parte das pessoas constituintes da rede social do indivíduo (Okun \& Lockwood, 2003), à crença de que ele é cuidado, amado, respeitado e valorizado, em função de pertencer a uma rede recíproca de comunicação (Cobb, 1976). O construto desempenha importante papel no enfrentamento de situações estressantes, ao permitir que os indivíduos satisfaçam suas necessidades e alcancem seus objetivos, mediante a administração dos recursos psicológicos e materiais adquiridos nas relações pessoais (Rodriguez \& Cohen, 1998). Nesse sentido, ele minimiza a incerteza, a ansiedade e a tensão, especialmente quando os indivíduos passam por problemas e crises (Lobburi, 2012), contribuindo, assim, para sua saúde física e mental (Rodriguez \& Cohen, 1998).

O referido construto pode ser de vários tipos, a depender dos recursos que são disponibilizados nas relações interpessoais (Rodriguez \& Cohen, 1998). Os tipos mais relatados na literatura são o emocional, o instrumental e o informacional. O suporte social emocional relaciona-se à atenção e à preocupação que um indivíduo demonstra pelo outro, por meio da comunicação verbal ou não verbal (Hogan, Linden, \& Najarian, 2002). O instrumental diz respeito ao apoio concreto que um indivíduo recebe do outro, a fim de satisfazer suas necessidades materiais (Seeman, 2008). O suporte social informacional, por fim, associa-se ao apoio fornecido por meio de informações (Seeman, 2008).

O suporte social pode ser ainda subdividido em percebido e recebido. O percebido consiste na percepção de que o suporte encontra-se disponível, caso o indivíduo dele precise, enquanto o recebido ocorre quando o indivíduo efetivamente recebe algum tipo de suporte (Cramer, Henerson, \& Scott, 1997). Focalizando especificamente o suporte social percebido, Zimet, Dahlem e Farley (1988) propõem que tal construto é de natureza multidimensional e se divide em três dimensões: apoio da família (o quanto o indivíduo percebe que recebe apoio dos membros de sua família), apoio de amigos (o quanto o indivíduo percebe que recebe apoio de seus amigos) e apoio de outro significativo (o quanto o indivíduo percebe que recebe apoio de outras pessoas que não sejam seus familiares ou amigos).

No que tange à avaliação do suporte social percebido, Sarason, Levine, Basham, e Saranson (1983) desenvolveram o Questionário de Apoio Social de Sarason, composto de 27 itens, com base no pressuposto de que o suporte social di- 
vide-se em dois elementos básicos: a percepção de que há pessoas disponíveis para quem se pode recorrer caso necessite; e o grau de satisfação com o apoio disponível (Sarason et al., 1983). O instrumento contempla, portanto, dois fatores: suporte social percebido e satisfação com o suporte social. Este instrumento foi traduzido e estudado no contexto brasileiro por Matsukura, Marturano e Oishi (2002). Os autores relatam que obtiveram um índice aceitável de fidedignidade teste-reteste e um alto índice de consistência interna (de 0,94 no teste e de 0,96 no reteste, em ambos os fatores).

Entre os instrumentos disponíveis para amostras brasileiras voltados à avaliação do suporte social percebido, com base na teoria de Rodriguez e Cohen (1998), que classifica o suporte social em emocional, instrumental e informacional, encontra-se a Escala de Percepção de Suporte Social (Siqueira, 2008), originalmente construída no Brasil. Por meio de procedimentos de análise fatorial exploratória, a autora verificou que a versão final da escala compôs-se de 29 itens, divididos em dois fatores (percepção de suporte social prático e emocional), com índices de consistência interna de 0,91 e 0,92 , respectivamente.

Outro instrumento disponível para o contexto brasileiro com fundamentação na teoria de Rodriguez e Cohen (1998) é a Escala de Percepção de Suporte Social - versão adulta (Cardoso \& Baptista, 2014), também construída originalmente para o contexto brasileiro. Os resultados da análise fatorial exploratória evidenciaram que a versão final da escala ficou composta por 36 itens, divididos em quatro dimensões: afetivo ( $\alpha$ $=0,92)$, interações sociais $(\alpha=0,75)$, instrumental $(\alpha=0,82)$ e enfrentamento de problemas $(\alpha$ $=0,83$ ).

Em síntese, no que diz respeito à avaliação do suporte social percebido, é possível encontrar na literatura nacional instrumentos originalmente desenvolvidos ou adaptados para amostras brasileiras. No entanto, um dos instrumentos mais utilizados no exterior para a avaliação do suporte social percebido é a Escala Multidimensional de Suporte Social Percebido (EMSSP)
(Zimet et al., 1988), que se apoia na premissa de que a percepção de suporte social provém de três fontes específicas, constituintes da rede social do indivíduo: família, amigos e outros significativos. De acordo com os autores, a divisão do construto em três fatores permite uma avaliação mais acurada da percepção do suporte social recebido pelo indivíduo, uma vez que as outras escalas não consideram a possibilidade de o suporte social emanar de fontes distintas.

O estudo de construção e de evidências de validade dos escores da EMSSP foi realizado com 275 estudantes universitários dos Estados Unidos (Zimet et al., 1988). Os resultados das análises fatoriais exploratórias levaram a uma versão final da escala composta por 12 itens, divididos nos três fatores teoricamente previstos: família, amigos e outros significativos. Os índices de consistência interna (alfa de Cronbach) desses três fatores foram, respectivamente, iguais a $0,87,0,85$ e 0,91 , com o instrumento total apresentando alfa de 0,88 .

A EMSSP vem tendo suas propriedades psicométricas testadas em diferentes países, como, por exemplo nos Estados Unidos (Aroian, Templin, \& Ramaswamy, 2010), em Portugal (Carvalho, Pinto-Gouveia, Pimentel, Maia, \& Mota-Pereira, 2011), na Tailândia (Wongpakaran, Wongpakaran, \& Ruktrakul, 2011) e na República do Malawi (Stewart, Umar, Tomenson, \& Creed, 2014). Em todos estes estudos, a escala teve sua estrutura de três fatores de primeira ordem confirmada, além de ter apresentado bons índices de consistência interna (alfas de Cronbach variando de 0,85 a 0,93 ). No entanto, o estudo de Mantuliz e Castillo (2002), com chilenos hipertensos, e o de Chou (2000), com adolescentes chineses, não replicaram a estrutura tri-fatorial, tendo obtido uma estrutura de dois fatores, com os itens do fator outros significativos agregados ao fator família.

Apesar de a escala ser uma das mais frequentemente usadas no exterior, a consulta à literatura nacional realizada em junho de 2015, nas bases de dados Scientific Electronic Library Online (SciELO) e Portal de Periódicos Eletrônicos de Psicologia (PePSIC), revelou 
que o instrumento ainda não possui evidências de validade de estrutura no contexto brasileiro. Justifica-se, assim, a realização de pesquisas adicionais destinadas a trazer evidências iniciais de validade dos escores da Escala Multidimensional de Suporte Social Percebido em amostras brasileiras. Essas investigações poderão disponibilizar aos investigadores brasileiros um instrumento de avaliação do suporte social percebido com possibilidades de ser futuramente adotado em investigações voltadas à análise das implicações do construto no bem-estar e qualidade de vida dos trabalhadores, uma vez que ele desempenha importante papel de proteção, ao reduzir o impacto de estressores do contexto organizacional (Carlson \& Perrewé, 1999). Assim, o presente trabalho pretendeu avaliar a estrutura interna, a consistência interna e a invariância dos parâmetros dos itens do instrumento em termos de gênero e categoria ocupacional (profissionais de áreas exatas e humanas).

A invariância de um instrumento constitui-se em técnica fundamental ao desenvolvimento e uso de instrumentos psicométricos, em função de permitir conclusões acerca da invariância da configuração e dos parâmetros do instrumento em diferentes grupos, o que irá garantir seu uso futuro nesses grupos (Cheung \& Rensvold, 2002). Fundamentando-se nessas considerações, hipotetizou-se que os itens da EMSSP seriam invariantes em função do gênero e da categoria ocupacional.

\section{Método}

\section{Participantes}

Participaram da pesquisa 831 trabalhadores de 25 estados brasileiros, mais o Distrito Federal, com o maior número de participantes distribuindo-se pelos estados de São Paulo (22,9\%) e Rio de Janeiro (16,5\%). A maior parte dos respondentes era do sexo feminino $(67,4 \%)$. No que tange à categoria ocupacional, $77,3 \%$ eram da área de humanas e $22,7 \%$, da área de exatas. Quanto à escolaridade, 68,6\% apresentavam pós-graduação lato ou stricto sensu, completa ou incompleta. A idade dos profissionais variou de 18 a 67 anos $(M=34,41 ; D P=10,73)$ e, o tempo de serviço, de 1 a 48 anos $(M=8,44 ; D P$ $=9,26$ ). O único critério de inclusão no estudo foi o fato de a pessoa estar trabalhando há pelo menos um ano, uma vez que uma das metas da pesquisa era disponibilizar aos pesquisadores brasileiros um instrumento de avaliação do suporte social percebido com possibilidades de ser futuramente adotado em investigações voltadas à análise das implicações do construto no bem-estar e qualidade de vida dos trabalhadores.

\section{Instrumento}

Para a mensuração do suporte social percebido, utilizou-se a Escala Multidimensional de Suporte Social Percebido (EMSSP), desenvolvida por Zimet et al. (1988). O instrumento compõe-se de 12 itens, divididos em três fatores (família, amigos, outros significativos), a serem respondidos por meio de escala Likert de sete pontos, variando de 'discordo muito fortemente' (1) a 'concordo muito fortemente' (7). Exemplo de item: Minha família tenta verdadeiramente me ajudar. Para a tradução da escala, foi adotado o procedimento de tradução e retradução (back-translation), que consiste em uma tradução dos itens para o português, seguida da tradução desta versão novamente para o inglês e da comparação dessas duas versões, a fim de verificar se houve uma equivalência conceitual (Borsa, Damásio, $\&$ Bandeira, 2012). Seguindo, portanto, algumas das recomendações desses autores, foi realizada, em uma primeira etapa, a tradução do instrumento original do idioma inglês para o português, por um pesquisador fluente em inglês. Na segunda etapa, a versão em português foi novamente traduzida para o inglês, por um professor de língua inglesa. $\mathrm{Na}$ etapa seguinte, por fim, dois pesquisadores da área de adaptação de escala, realizaram uma revisão técnica e a avaliação da equivalência semântica das duas versões em inglês. O instrumento de coleta de dados contou, ainda, com perguntas destinadas a levantar informações sociodemográficas dos participantes, no que diz respeito à idade, ao sexo, à escolaridade, à profissão, ao estado civil, ao tempo de serviço e ao estado em que viviam. 


\section{Procedimento de Coleta de Dados}

A coleta de dados foi realizada em versão online. Um formulário foi criado no aplicativo Google Docs. Os indivíduos foram convidados a participar por meio de mensagens em dois sites de relacionamento: o Facebook e o Linkedin, nas quais era enviado o link para o questionário.

\section{Procedimento de Análise dos Dados}

Para avaliar a dimensionalidade dos escores da EMSSP, os dados foram analisados por meio da análise paralela (com 200 reamostragens; e percentil 95 dos eigenvalues aleatórios), da Exploratory Graph Analysis (EGA; Golino \& Epskamp, 2017) e da comparação entre modelos rivais por meio da modelagem por equações estruturais. A EGA é uma técnica desenvolvida recentemente, que utiliza a análise de redes aliada a algoritmo para a detecção de fatores subjacentes aos dados. Essa técnica apresenta acurácia superior à análise paralela na estimação do número verdadeiro de fatores, principalmente para estruturas com altas correlações entre os fatores e baixo número de itens por fator (Crawford et al., 2010; Golino \& Epskamp, 2017; Ruscio \& Roche, 2012).

Para as análises fatoriais confirmatórias, os parâmetros dos itens e dos participantes foram estimados por meio de weighted least square mean and variance ajusted (WLSMV), declarando os itens como variáveis categóricas-ordinais. Foram analisados os seguintes índices de ajuste, atendendo-se às recomendações de Byrne (2012): qui-quadrado (testa a diferença entre a matriz empírica e a matriz do modelo teórico, sendo que quanto maior o valor do $\chi^{2}$ pior o ajustamento); Root-Mean-Square Error of Approximation (RMSEA - deve se situar abaixo de 0,08 , aceitando-se valores até 0,10$)$; Tucker-Lewis Index (TLI - são aceitáveis modelos com valores acima de 0,95); Comparative fit index (CFI - são aceitáveis valores acima de 0,95$)$. O poder estatístico foi estimado por meio de estudos de simulação Monte Carlo, conforme sugerido por Muthén e Muthén (2002). Nesse contexto, foram avaliados os seguintes critérios: vieses de parâmetros menores que 10\% (i.e. [parâmetro - média dos parâmetros simulados] / parâmetro), vieses dos erros de estimação menores que 5\%; coverage acima de 0,90 (o valor de coverage indica a proporção de replicações nas quais o IC de 95\% contém o valor verdadeiro do parâmetro).

A consistência interna do instrumento foi calculada por meio do alfa de Cronbach, da variância média extraída e da confiabilidade composta. Para avaliar a invariância dos parâmetros dos itens entre os grupos separados por sexo e área de formação acadêmica, utilizou-se a modelagem de equações estruturais multigrupos (Damásio, 2013). Para tanto, foram testados modelos nos quais foram fixados o número de itens e fatores (invariância configural), as cargas fatoriais (invariância métrica) e os thresholds e scalars (invariância escalar). As diferenças entre os ajustes dos modelos foram avaliadas por meio da diferença do qui-quadrado $\left(\Delta \chi^{2}\right)$ e do CFI ( $\Delta \mathrm{CFI}$ ). Os dados foram analisados por meio dos softwares Mplus (v. 7.11) e R.

\section{Procedimentos Éticos}

A pesquisa foi submetida ao Comitê de Ética em Pesquisa da instituição dos autores, tendo recebido o número de parecer de 689.286. A concordância em participar da pesquisa foi obtida por meio do Termo de Consentimento Livre e Esclarecido.

\section{Resultados}

\section{Análise da Estrutura e Consistência Interna}

A literatura sobre a estrutura da escala apresenta evidências de modelos com duas e três dimensões. Nesse sentido, buscou-se avaliar a dimensionalidade dos escores, primeiramente, por meio de uma análise paralela. Tal método indicou a extração de duas dimensões (quatro primeiros eigenvalues: empíricos 7,13 / 1,88 / $0,87$ / 0,37; aleatórios $1,25 / 1,18 / 1,38 / 1,1)$. Nesse contexto, o modelo aglutinaria as dimensões Família e Outros Significativos e manteria a dimensão original Amigos.

No entanto, a EGA indicou a extração de três dimensões (Figura 1). No gráfico, a espes- 
sura da linha que conecta duas variáveis indica a força da relação. Assim, percebe-se na Figura 1 , que as relações internas, entre os itens de cada dimensão, são maiores do que as associações com os itens das demais dimensões. Tal resultado aponta para três dimensões distintas.

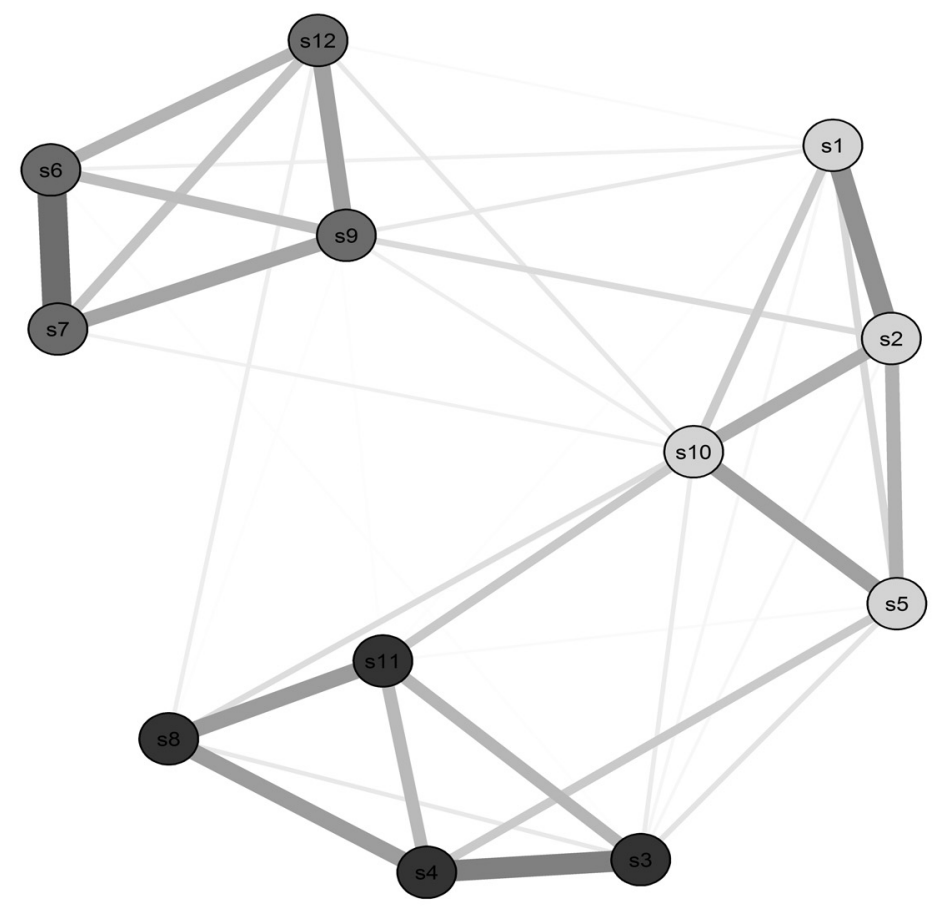

$\begin{array}{ll}\because & 1 \\ \circ & 2 \\ - & 3\end{array}$

Figura 1. Exploratory Graphic Analysis.

Para ampliar a discussão da dimensionalidade, por meio da modelagem por equações estruturais, buscou-se testar o ajuste aos dados dos seguintes modelos: duas dimensões, conforme configuração dos itens e fatores evidenciados por Chou (2000) e Mantuliz e Castillo (2002); três dimensões, conforme o modelo original da escala (Zimet et al., 1988). Os resultados eviden- ciaram que o modelo de três fatores correlacionados (família, amigos e outros significativos), da escala original de Zimet et al. (1988), foi o que apresentou melhores índices de ajuste, em comparação ao modelo de dois fatores (família-outros significativos e amigos) (Tabela 1). A Figura 2 traz a representação gráfica do modelo de três fatores e as cargas fatoriais padronizadas dos itens.

Tabela 1

Análise Fatorial Confirmatória da Escala Multidimensional de Suporte Social Percebido

\begin{tabular}{ccccc}
\hline Modelos & $\chi^{2}(g l)$ & CFI & TLI & RMSEA \\
\hline Dois fatores & $12161,41(53)$ & 0,966 & 0,957 & 0,166 \\
Três fatores & $405,21(51)$ & 0,987 & 0,990 & 0,091 \\
\hline
\end{tabular}

Notas. $\chi^{2}=$ qui-quadrado; $g l=$ graus de liberdade; TLI $=$ Tucker Lewis Index; $\mathrm{CFI}=$ Comparative Fix Index; RMSEA $=$ Root Mean Square Error of Approximation. 


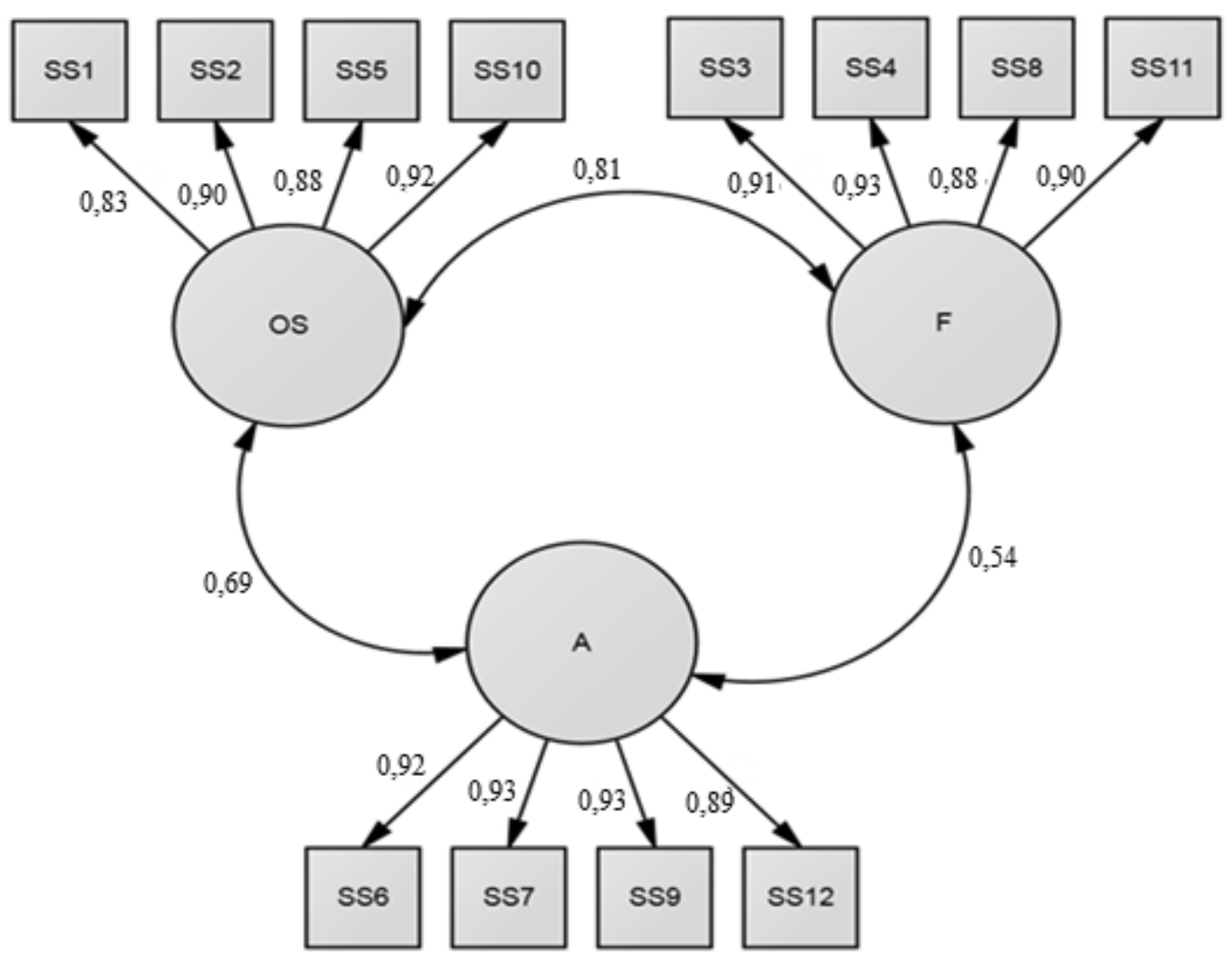

Figura 2. Modelo Final da Escala Multidimensional de Suporte Social Percebido, com as cargas fatoriais padronizadas.

Notas. OS = Outros Significativos; F = Família; A = Amigos; $\mathrm{SS}=$ Suporte social.

No que se refere à validade discriminante dos escores latentes, o modelo apresentado na Figura 2 indicou alta correlação $(0,81)$ entre as dimensões Outros Significativos e Família. Tal correlação poderia ser um indício de ausência de validade discriminante. Contudo, observaram-se cargas fatoriais altas entre os itens dessas dimensões (média das cargas fatoriais: Outros Significativos $=0,88 ;$ Família $=0,91)$ e maiores do que a correlação entre as dimensões latentes. Tais resultados indicam que a maior parte da variância dos itens explicada pelo modelo (i.e. desconsiderando o erro de medida) pode ser atribuída aos fatores latentes e não à correlação entre os fatores. Assim, os resultados do presente estudo evidenciam que as dimensões Outros Significativos e Família são construtos distintos (validade discriminante), embora altamente correlacionados.

A versão brasileira da Escala Multidimensional de Suporte Social Percebido ficou, portanto, composta de três fatores, com quatro itens cada. Ademais, os indicadores de variância média extraída foram iguais a 0,84 (amigos), 0,82 (família) e 0,78 (outros significativos); e as confiabilidades compostas foram iguais a 0,96 (amigos), 0,95 (família) e 0,93 (outros significativos). Os índices de consistência interna dos fatores da escala, calculados pelo alfa de Cronbach, foram iguais a 0,93 (amigos), 0,91 (família) e 0,90 (outros significativos). Tais resultados indicam que os escores dos três fatores podem ser estimados com alta precisão, mesmo com a quantidade reduzida de itens.

Foram realizadas, ainda, simulações com bancos de dados de 831 sujeitos e, em todas elas, os valores de vieses das cargas fatoriais e das correlações entre os fatores foram inferiores a 0,001 ; os vieses dos erros de estimação variaram de 0 a 0,02; os valores de coverage variaram de 0,94 a 0,95 . Tais resultados indicam que a estimação dos parâmetros do modelo foi realizada com poder estatístico acima de 0,80 . 


\section{Análise de Invariância}

Considerado o modelo de estrutura de três dimensões de primeira ordem encontrado, buscou-se, por meio da análise fatorial multigrupo
(AFCMG), avaliar a invariância dos parâmetros dos itens entre os participantes do gênero masculino e feminino, bem como entre trabalhadores das áreas de exatas e de humanas. Os resultados da AFCMG são indicados na Tabela 2.

Tabela 2

Análise Fatorial Confirmatória Multigrupo (AFCMG) para a Variável Sexo e Categoria Ocupacional

\begin{tabular}{ccccc}
\hline $\begin{array}{c}\text { Gênero } \\
\text { (homens x mulheres) }\end{array}$ & $\chi^{2}(g l)$ & CFI & TLI & RMSEA \\
\hline Configural & $601,46(102)$ & 0,986 & 0,989 & 0,109 \\
Métrica & $594,62(111)$ & 0,987 & 0,989 & 0,102 \\
Escalar & $601,86(168)$ & 0,993 & 0,991 & 0,079 \\
Categoria & $\chi^{2}(g l)$ & CFI & TLI & RMSEA \\
(exatas x humanas) & $649,34(102)$ & 0,984 & 0,988 & 0,114 \\
Configural & $615,70(111)$ & 0,987 & 0,989 & 0,105 \\
Métrica & $631,46(168)$ & 0,992 & 0,990 & 0,081 \\
\hline
\end{tabular}

Notas. $\chi^{2}=$ qui-quadrado; $g l=$ graus de liberdade; TLI $=$ Tucker Lewis Index $; \mathrm{CFI}=$ Comparative Fix Index RMSEA $=$ Root Mean Square Error of Approximation; Configural = estrutura fatorial fixa entre os grupos; Métrica =estrutura fatorial e cargas fatoriais fixas entre os grupos; Escalar =estrutura fatorial, cargas fatoriais, thresholds e scalars fixos entre os grupos. $n$ homens $=271 ; n$ mulheres $=560 ; n$ exatas $=189 ; n$ humanas $=642$.

Em relação à invariância dos parâmetros entre os grupos do gênero masculino e feminino, as imposições de restrições trouxeram diferenças pequenas e praticamente desprezíveis nos indicadores analisados. Salienta-se que as diferenças dos qui-quadrados entre os modelos mais restritos e os menos restritos não foram estatisticamente significativas (i.e. $\Delta \chi^{2} / \Delta g l<1,96$ ), assim como as diferenças de CFI foram inferiores a 0,01 . Tais resultados indicam que, para o modelo de três fatores de primeira ordem, as cargas fatoriais, os thresholds e scalars foram invariantes entre os gêneros masculino e feminino (Cheung \& Rensvold, 2002).

No que tange aos trabalhadores das áreas de ciências humanas e de ciências exatas, os resultados também demonstraram invariância configural, métrica e escalar do instrumento, uma vez que as diferenças acarretadas pelas restrições foram praticamente desprezíveis e não foram estatisticamente significativas. Nesse sentido, os parâmetros dos itens da Escala Multidimensional de Suporte Social Percebido não são enviesados pela variável área de atuação. No que se refere ao poder estatístico, mesmo para os bancos simulados com 189 sujeitos (tamanho de amostra igual ao menor grupo investigado - ciências exatas), o poder estatístico foi superior a 0,80 , numa evidência de que os diferentes tamanhos dos grupos adotados na análise da invariância fatorial não interferiram nos resultados obtidos.

\section{Discussão}

O presente trabalho teve como objetivo investigar as evidências de validade de estrutura interna da Escala Multidimensional de Suporte Social Percebido, bem como sua consistência interna e a invariância dos parâmetros dos itens. Os resultados da EGA e da AFC apontaram para adequação de um modelo de três dimensões. $\mathrm{O}$ resultado contraditório da análise paralela, que indicou a extração de apenas duas dimensões, pode ser explicado pela limitação desse método, 
que tende a subestimar o número de dimensões de modelos cujas correlações entre os fatores são altas e o número de itens por fator é baixo (Crawford et al., 2010; Golino \& Epskamp, 2017; Ruscio \& Roche, 2012).

Ressalta-se que o modelo de três fatores de primeira ordem avaliado pela AFC foi configurado com base na estrutura teórica original do instrumento, mantendo o caráter hipotético-dedutivo dessa análise. Assim, o modelo confirma a estrutura original do instrumento (Zimet et al., 1988), bem como é compatível com os resultados de outros estudos de evidencias de validade dos escores (Aroian et al., 2010; Carvalho et al., 2011; Stewart et al., 2014; Wongpakaran et al., 2011). O modelo de três fatores mostra-se, ainda, congruente com a teoria de Zimet et al. (1988), segundo a qual o construto suporte social percebido é de natureza multidimensional, na medida em que o apoio social é proveniente de diferentes fontes da rede social do indivíduo.

No entanto, as investigações de Mantuliz e Castillo (2002), em uma amostra de chilenos hipertensos, e de Chou (2000), em pesquisa com adolescentes chineses, não replicaram a estrutura trifatorial, tendo, ao contrário, obtido uma estrutura de dois fatores para a escala. Na presente pesquisa, porém, tal modelo de dois fatores apresentou ajuste aos dados inferior ao modelo original de três dimensões. Nesse sentido, a presente pesquisa adiciona evidências de que o modelo verdadeiro para a escala está mais próximo de três dimensões do que do modelo de dois fatores.

Cumpre destacar, porém, que tal modelo de estrutura com três fatores apresentou correlação alta entre as dimensões Família e Outros Significativos, porém menor do que as médias das cargas fatoriais dessas mesmas dimensões. A correlação mais alta observada entre essas duas dimensões converge com os resultados de outros estudos (Bruwer, Emsley, Kidd, Lochner, \& Seedat, 2008; Wongpakaran et al., 2011), muito embora neles também a solução de três fatores tenha apresentado melhor ajuste aos dados. Tal resultado pode se dever ao fato de o significado de família como fonte de suporte provavelmente mudar ao longo do ciclo vital, com os indivíduos que já possuem suas próprias famílias passan- do a vê-las também como a principal fonte de referência, no que concerne às pessoas que lhes são significativas (Zimet et al., 1988). De todo modo, tais asserções necessitam ser confirmadas em estudos futuros.

Os indicadores de precisão VME, CC e alfa de Cronbah apresentaram valores altos. Especificamente no que se refere ao alfa de Cronbach, os resultados evidenciados na presente pesquisa são semelhantes aos observados em diferentes contextos, como nos Estados Unidos (Zimet et al., 1988), em Portugal (Carvalho et al., 2011), na Tailândia (Wongpakaran et al., 2011) e na República do Malawi (Stewart et al., 2014). De modo similar, o alfa de Cronbach encontrado no fator geral também foi semelhante ao de outros estudos (Carvalho et al., 2011; Stewart et al., 2014; Wongpakaran et al., 2011; Zimet et al., 1988). Tais resultados constituem uma indicação de que os escores da escala costumam apresentar índices adequados de precisão em amostras de nacionalidades diferentes.

Os resultados das análises fatoriais confirmatórias multigrupo (AFCMG) demonstraram que a escala não apresentou vieses de resposta para homens e mulheres, nem tampouco para trabalhadores das área de ciências exatas e de ciências humanas. Pode-se afirmar, portanto, que os escores de suporte social percebido obtidos por meio do instrumento são invariantes para esses grupos, que podem, assim, ser comparados entre si. Tais resultados também podem ser vistos como um indicador de que os parâmetros dos itens da escala são estáveis em amostras com perfis distintos.

\section{Considerações Finais}

Os resultados do presente estudo indicam que a Escala Multidimensional de Suporte Social Percebido apresentou evidências de validade de estrutura interna, bem como consistência interna adequada e invariância dos parâmetros dos itens. No entanto, cumpre registrar as limitações do estudo. Uma delas é a de que o instrumento utilizado foi de autorrelato e nem sempre o que é relatado pelos participantes condiz com a realidade. Outrossim, a maioria da amostra estava 
concentrada em uma única região do Brasil, o sudeste, o que dificulta a generalização dos resultados para as demais regiões brasileiras. Por fim, o número de participantes do grupo da área de humanas foi bem superior ao da área de exatas, assim como o número de participantes do sexo feminino foi maior do que o de participantes do sexo masculino. Ressalta-se, no entanto, que em todos os grupos o poder estatístico foi superior a 0,80 .

No que diz respeito a uma agenda futura de pesquisas, sugere-se a realização de estudos destinados a aprofundar a rede nomológica do suporte social percebido, em especial no que se refere ao impacto dos fatores família, amigos e outros significativos no bem-estar e qualidade de vida dos trabalhadores. Estas pesquisas poderiam ser de natureza longitudinal, a fim de se obter maior compreensão acerca das relações entre essas variáveis. Sugere-se, ainda, que sejam realizadas pesquisas comparativas com populações com outras características, como, por exemplo, adolescentes e adultos, que provavelmente apresentam percepções de suporte social diferenciadas. Em que pesem as limitações mencionadas, os achados ora obtidos permitem a conclusão de que a versão brasileira da Escala Multidimensional de Suporte Social Percebido mostrou-se um instrumento apropriado à avaliação da percepção que o indivíduo tem do apoio social recebido da família, dos amigos e de outros significativos, em amostras brasileiras.

\section{Referências}

Aroian, K. J., Templin, T. N., \& Ramaswamy, V. (2010). Adaptation and psychometric evaluation of the Multidimensional Scale of Perceived Social Support for Arab immigrant women. Health Care for Women International, 31(2), 153-169. doi:10.1080/07399330903052145

Borsa, J. C., Damásio, B. F., \& Bandeira, D. R. (2012). Adaptação e validação de instrumentos psicológicos entre culturas: Algumas considerações. Paidéia (Ribeirão Preto), 22(53), 423-432. doi:10.1590/S0103-863X2012000300014

Bruwer, B., Emsley, R., Kidd, M., Lochner, C., \& Seedat, S. (2008). Psychometric properties of the Multidimensional Scale of Perceived So- cial Support in youth. Comprehensive Psychiatry, 49(2), 195-201. doi:10.1016/j.comppsych.2007.09.002

Byrne, B. M. (2012). Structural equation modeling with Mplus: Basic concepts, applications, and programing. New York: Routledge.

Cardoso, H. F., \& Baptista, M. N. (2014). Escala de Percepção de Suporte Social (versão adulta) EPSUS-A: Estudo das qualidades psicométricas. Psico-USF, 19(3), 499-510. doi:10.1590/141382712014019003012

Carlson, D. S., \& Perrewé, P L. (1999). The role social support in the stressor-strain relationship: An examination of work-family conflict. Journal of Management, 25(4), 513-540. doi:10.1177/014920639902500403

Carvalho, S., Pinto-Gouveia, Pimentel, P., Maia, D., \& Mota-Pereira, J. (2011). Características psicométricas da versão portuguesa da Escala Multidimensional de Suporte Social Percebido (Multidimentional Scale of Perceived Social Support - MSPSS). Psychologica, (54), 331-357. doi:10.14195/1647-8606_54_13

Cheung, G. W., \& Rensvold, R. B. (2002). Evaluating goodness-of-fit indexes for testing measurement invariance. Structural Equation Modeling, 9(2), 233-255. doi:10.1207/S15328007SEM0902_5

Chou, K. L. (2000). Assessing Chinese adolescents' social support: The Multidimensional Scale of Perceived Social Support. Personality and Individual Differences, 28(2), 299-307. doi:10.1016/S01918869(99)00098-7

Cobb, S. (1976). Social support as a moderator of life stress. Psychosomatic Medicine, 38(5), 300-314. doi:10.1097/00006842-197609000-00003

Cramer, D., Henderson, S., \& Scott, R. (1997). Mental health and desired social support: A four-wave panel study. Journal of Social and Personal Relationships, 14(6), 761-775. doi:10.1177/0265407597146003

Crawford, A. V., Green, S. B., Levy, R., Lo, W. J., Scott, L., Svetina, D., \& Thompson, M. S. (2010). Evaluation of parallel analysis methods for determining the number of factors. Educational and Psychological Measurement, 70, 885901. doi:10.1177/0013164410379332

Damásio, B. F. (2013). Contribuições da análise fatorial confirmatória multigrupo (AFCMG) na avaliação de invariância de instrumentos 
psicométricos. Psico-USF, 18(2), 211-220. doi:10.1590/S1413-82712013000200005

Golino, H. F., \& Epskamp, S. (2017). Exploratory graph analysis: A new approach for estimating the number of dimensions in psychological research. Plos One. doi:https://doi.org/10.1371/ journal.pone.0174035

Hogan, B., Linden, W., \& Najarian, B. (2002). Social support interventions: Do they work? Clinical Psychology Review, 22(3), 381-440. doi:10.1016/s0272-7358(01)00102-7

Lobburi, P. (2012). The influence of organizational and social support on turnover intention in collectivist contexts. Journal of Applied Business Research, 28(1), 93-104. doi:10.19030/jabr. v28i1.6687

Mantuliz, M., \& Castillo, C. (2002). Validación de una escala de apoyo social percibido en un grupo de adultos mayors adscritos a un programa de hypertension de la region metropolitan. Ciencia y Enfermeria, 8(1), 49-55. doi:10.4067/S07175532002000100007

Matsukura, T. S., Marturano, E. M., \& Oishi, J. (2002). O questionário de suporte social (SSQ): Estudos de adaptação para o português. Revista Latino-Americana de Enfermagem, 10(5), 675 681. doi:10.1590/S0104-11692002000500008

Muthén, L. K., \& Muthén, B. O. (2002). How to use a Monte Carlo study to decide on sample sizes and determine power. Structural Equation Modeling, 9(4), 599-620. doi:10.1207/ S15328007SEM0904 8

Okun, M. A., \& Lockwood, C. M. (2003). Does level of assessment moderate the relation between social support and social negativity? A meta-analysis. Basic and Applied Social Psychology, 25(1), 15-35. doi:10.1207/ S15324834BASP2501 2

Rodriguez, M. S., \& Cohen, S. (1998). Social support. Encyclopedia of Mental Health, 3, 535-544. Retrieved from http://www.psy.cmu.edu/ scohen/ socsupchap98.pdf

Ruscio, J., \& Roche, B. (2012). Determining the number of factors to retain in an exploratory factor analysis using comparison data of known factorial structure. Psychological Assessment, 24(2), 282-292. doi:10.1037/a0025697
Sarason, I. G., Levine, H. M., Basham, R. B., \& Sarason, B. R. (1983). Assessing social support: The social support questionnaire. Journal of Personality and Social Psychology, 44(1), 127-139. doi:10.1037//0022-3514.44.1.127

Seeman, T. (2008). Social \& social conflict: Section one - Social support. Retrieved from http:// www.macses.ucsf.edu/research/psychosocial/ socsupp.php.

Siqueira, M. M. M. (2008). Construção e validação da Escala de Percepção de Suporte Social. Psicologia em Estudo, 13(2), 381-388. Recuperado em http://www.scielo.br/pdf/pe/v13n2/a21v13n2

Stewart, R. C., Umar, E., Tomenson, B., \& Creed, F. (2014).Validation of the multi-dimensional scale of perceived social support (MSPSS) and the relationship between social support, intimate partner violence and antenatal depression in Malawi. BioMed Central Psychiatry, 14(1), 1-11. doi:10.1186/1471-244X-14-180

Wongpakaran, T., Wongpakaran, N., \& Ruktrakul, R. (2011). Reliability and validity of the Multidimensional Scale of Perceived Social Support (MSPSS): Thai version. Clinical Practice \& Epidemiology in Mental Health, 7, 161-166. doi:10.2174/1745017901107010161

Zimet, G. D., Dahlem, N., Zimet, S., \& Farley, G. (1988). The Multidimensional Scale of Perceived Social Support. Journal of Personality Assessment, 52(1), 30-41. doi:10.1207/ s15327752jpa5201_2
Recebido: $27 / 04 / 2016$

$1^{a}$ revisão: 04/05/2016

$2^{a}$ revisão: 03/10/2016

Aceite final: 11/10/2016 